\title{
Hybrid Framework for Web Services on Android Mobile Host
}

\author{
Kishor Wagh \\ AISSMS IOIT, Computer Engineering Department \\ Email:waghks@gmail.com
}

Received: $2^{\text {th }}$ December 2018, Accepted: $13^{\text {th }}$ February 2019, Published: $3^{\text {th }}$ June $^{2019}$

\begin{abstract}
Growth in Mobile hardware manufacturing and Mobile data services, increases the use of mobile phones in day to day activities for personal work or business transactions. Increased use of mobile phones and mobile connectivity with web services is the new domain in information systems research. The smart phone is a true ubiquitous computing device provides a wide range of services to any time and any place. This paper introduces the novel framework for provisioning web services for smart phones with special focus on access web services through SMS messaging service as well as the HTTP protocol at our mobile Host.
\end{abstract}

Keywords

Android; SOAP; REST; Mobile Web Services; Hybrid Framework

\section{Introduction}

Today is the era of Internet of Things (IoT). The aim of Internet of Things is to connect anything to the Internet. Anything means any device, any valuable thing or important thing such as car key, lucky pen, etc. Internet of Thing enables users to access services anywhere, anytime from any location. This is possible only through Internet, web applications or web services. In future we can see different web services instead of a standalone application. For example, instead of payroll system, it will be payroll service. These services are accessed by users through the Internet. Same time unbelievable development in area of mobile manufacturing, wireless technologies and reduced cost of mobile smart phones and Internet data plans. New wireless communication standards such as Long Term Evolution (LTE) and LTE-Advanced increases data transfer speed, and new polices of IT industry is Bring Your Own Device (BYOD) increasing the use of mobile devices.

Uses of mobiles are increasing every second. As per market research by Cisco Visual Networking Index and International Data Corporation use of mobile devices increased every year [2, 3].

From above prediction, smart phones will play a central role in daily activities at home and business places. Mobile phones are used as a web client to access web services from a web service provider, but it changes its role form web service consumer to web service provider and enables us to deploy web services on mobile host. To provide efficient, reliable, lightweight framework to fulfill end user requirements without affecting its basic functionality for voice call. Web Service technology is the predecessor of distributed computing; its technologies are Common Object Request Broker Architecture (CORBA) S. Vinoski [4], Remote Method Invocation (RMI) J. Maassen et al. [5], Distributed Component Object Model (DCOM) F. Plil et al. [6]. These technologies dont provide a generic web solution to the user, to satisfy their needs, so web services are used. Web Services primary goal is to interrelate distributed functionality for platform independent. But unlike its predecessors, it achieves its goal in an elegant and technology-neutral manner. It has capability to provide interface for independent to operating system, programming language and hardware.

A web service provisioning more challengeable in wireless environment as compared to wired network. There are two types of web services are used as Simple Object Access Protocol (SOAP) and Representational State Transfer (REST). In Paper [26, 1] author expanded the web service provisioning on android mobile host and objective of study is to use mobile host anywhere, anytime. Hosting emergency health related web services on mobile android host is new, so in its early stage to investigate hybrid framework that support's open source Android environment, and provide access to web services from any mobile through SMS messaging or web browsers. The framework provides an alternative way to access web services from mobile host.

Provisioning web services on mobile phone enrich researchers to provide better solution those to who are far away from urban area and even rural area people can access web services without mobile internet through Short Message Services (SMS). In this study, we have analyzed the feasibility of a mobile host in android environment and provide access of web service through HTTP with mobile internet and SMS with without mobile Internet. Hosting web services on smart phones increases the scalability and usability of web services to the mobile web service provisioning domain. The paper discusses Provisioning of Web Services on Android mobile device in dynamic environment, with its challenges of wireless network and mobile device.

This paper is organized as follows: section 2 discusses the basic paradigms of mobile web service provisioning and its architectures. Section 3 describes the proposed hybrid framework for mobile host and 
performance evaluation model. Section 4 describes the details of test setup used for experimentation. Section 5 discusses results of REST based hybrid framework. Section 6 enlists the related work of mobile web service provisioning domain and last section concludes the paper with possible future research directions

\section{Related Work}

S. N. Srirama [17, 13, 18, 19] was studied provisioning of Mobile Web Services Mediation Framework for mobile Peer-To -Peer network. It is based on kSOAP and supports the publishing and discovery of services. Yeon-Seok Kim and Kyong-Ho Lee. [20, 21] elaborated migration based lightweight framework for mobile web services. The proposed framework has functionalities to e process of SOAP messages, the execution and migration of services, the management of service directory, and the publishing and discovery of services. In this framework mobile host migrates services, when its battery goes down. M. Asif and S. Majumdar. [22, 23] described Partitioning frameworks for mobile web service provisioning. Author offloaded Web Service partitions using three types of partitioning frameworks. The first framework uses an intermediate node to intercept the requests from a Web Service client to a mobile device and processes it partly before forwarding it to the Web Service provider running on the mobile device. The second framework is based on a backend node for execution of offloaded parts of web service. The backend node is used to execute offloaded partitions of web service on request for the Web Service provider (mobile node) and send the result back to the Web Services provider that uses it to satisfy the client request. The third framework uses a forwarding node. The forwarding node is used to execute offloaded partitions of an application, aggregate overall results and forward the response to the Web Service client instead of sending the result back to the mobile device. K. Elgazzar et al. [24, 25] proposed framework was able to provide personalized SOAP based Web services for efficient Web services provisioning in mobile heterogeneous environments with resourceconstrained mobile devices. The framework provides ubiquitous wireless access, multi-homed interfaces, their attachment to specific context information such as user and location, and their capability to behave in an ad-hoc manner. The framework was able to provide reliable and personalized Web services while maintaining the service availability.

Overview of Mobile Web Services

The main architectural paradigm designs supported by mobile web service provisioning are the SOA and ROA [7, 8, 9, 10, 13, 14]. Web service technologies and standards began to emerge around 2000 to overcome the problems in distributed technologies, remove the complexities and provide easy generic solution to work with any platform. Web services are a realization of the SOA and ROA paradigm with the existing Internet and World Wide Web technologies like Hyper Text Transport Protocol (HTTP), SOAP and the eXtensible Markup Language (XML). HTTP is used as the transport mechanism for XML based service base message exchange. HTTP is the primary transport protocol for today's web services instead of SOAP over HTTP. Mobile Web services are based on two architectural concepts: SOAP and REST [11, 12]. The comparison between SOAP and REST are shown in table 1:

\begin{tabular}{|l|l|l|}
\hline Features & Soap Based Web Services [15] & REST Based Web Services [7, 16] \\
\hline Architectural Style & Service centric & Resource centric \\
\hline Coupling Supported & Tightly Coupling & Loose Coupling \\
\hline Transport Protocol & HTTP, SMTP, TCP, UDP, or JMS & HTTP \\
\hline Web Services Access & Single end point access to web services & $\begin{array}{l}\text { URI for each resource so many ways to } \\
\text { access web services }\end{array}$ \\
\hline QoS & WS specifications & Transport dependable \\
\hline Interface & Separate interface for each service & Web Browser based interface \\
\hline Invocation & RPC, HTTP, SMTP & HTTP \\
\hline Message Payload & Heavy & Light \\
\hline Data Representation & XML & XML, JSON \\
\hline Scalability & Less & More \\
\hline Security & WS-Security & HTTP Security \\
\hline Suitability & Less Suitable for Mobile Devices & More Suitable for Mobile Devices \\
\hline
\end{tabular}

Table 1: Comparison between SOAP and REST Based Web Services

\section{Proposed Hybrid Framework for Mobile Host}

Traditional web service provisioning frameworks are based on SOAP concept and for mobile host used as middleware between web service client and high end system. It was not full-fledged mobile host, it was the 
only web service handler, when web clients request for a web service to a mobile host. Mobile host only forwards the request to the high end web service provider. The web service provider is a high end machine which processes the mobile host request and generates the response and generated response forwarded to the mobile host and the mobile host sends the response to the web service client.

The Proposed RESTful based hybrid framework would provide better solutions and reduce the burden on mobile device resources, would offer reliable services that serve the best interest of users, in addition to support, alternatively access web services through SMS messaging from any mobile device means any platform, without enabling data services and non-Java mobile devices. SOAP is the de facto standards for web services and SOAP based frameworks are used by Web service provisioning. Proposed framework is works on resource constraints devices and gives good results as compared to traditional frameworks.

We implemented hybrid framework for mobile host based on REST architectural concept. Mobile host is a full-fledged web service provider not a web client handler and it is accessed through HTTP as well as SMS (Short Messaging Service) as shown in figure 1. SMS provides an alternative way to access Mobile host, which allows the web service client to access web services without any wireless internet service on the web service client.

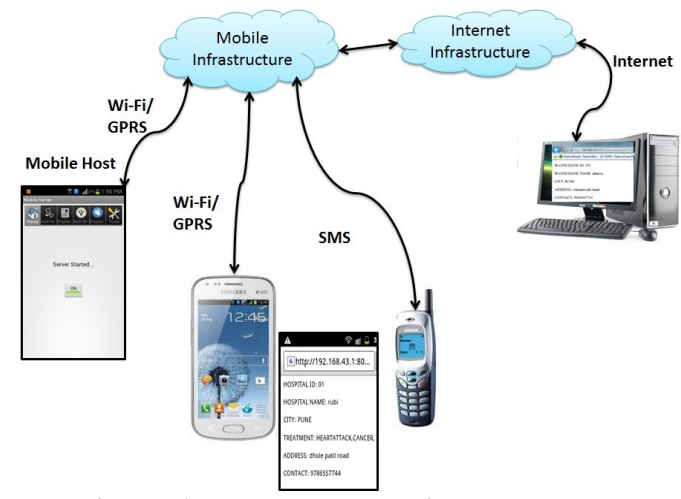

Figure 1: Proposed Hybrid Framework

The proposed hybrid framework is based on deployment scenario of the mobile host connected to mobile infrastructure through any wireless internet service. The mobile web service clients are also connected through any wireless internet service to access web service through the browser using the HTTP protocol and those mobile clients have no Internet they can access web services through SMS. Fixed wired web service clients access web services through a browser using HTTP protocol.

\section{Testing the Hybrid Framework}

Performance of Hybrid Framework is extensively and rigorously tested. The hospital and blood bank search engine named mHealth Care Service is used for evaluation of the proposed architecture. It provides 3 static and 2 dynamic HTML server response pages that can be used to provide responses to client requests. The names, descriptions and response type of the HTML pages are listed in the table 2.

\begin{tabular}{|l|l|l|}
\hline File Name & Description & Response Type \\
\hline home.html & $\begin{array}{l}\text { Contains two buttons for the hospital and blood bank, } \\
\text { which redirects to selected web service }\end{array}$ & Static \\
\hline hospital.html & $\begin{array}{l}\text { Specifies selection criteria of hospitals based on 1) City name 2) } \\
\text { Disease Static }\end{array}$ & Static \\
\hline bloodbank.html & Specifies selection criteria of blood banks based 1) City name & Static \\
\hline hspresonse.html & $\begin{array}{l}\text { Generate a response to client requests, i.e. get number of hospitals } \\
\text { which fulfil the selection criteria. Response type of this page is } \\
\text { dynamic because number of hospitals selected is depending on the } \\
\text { city name and disease name }\end{array}$ & Dynamic \\
\hline bbresponse.html & $\begin{array}{l}\text { Generate response with a number of blood banks, which fulfil the } \\
\text { selection criteria. Response type of this page is dynamic because } \\
\text { number of blood banks selected is depending on the city name }\end{array}$ & Dynamic \\
\hline
\end{tabular}

TEST Setup

Table 2: HTML Pages for Accessing Web Services through Client

For performance evaluation of Hybrid framework following test setup used:

- Mobile Host (Service Provider): Host 1: Samsung Galaxy S Duos S7562, 4GB internal memory, $1 \mathrm{GHz}$ Cortex-A5 processor, Host 2: HTC Explorer A310E. Host 3: Samsung Galaxy S3 and Host 4: Moto g. 
- Web service clients: Mobile phone, Tablet, Laptop, PC, Notebook

- Development Environment: Eclipse

- Database used: SQLite

- Communication Environment: Wi-Fi, 2G, 3G, 4G/LTE and SMS

- Web Services: Hospital Web Service, Blood Bank Web Service

\section{Results and Discussion}

Figure 2 shows the graphical representation between payload size generated by SOAP based framework and REST based Hybrid framework for Hospital search and Blood bank search web service. Payload generated by SOAP based traditional framework is more as compared to REST based Hybrid Framework. The small payload means less processing time and less processing time means less battery consumption.

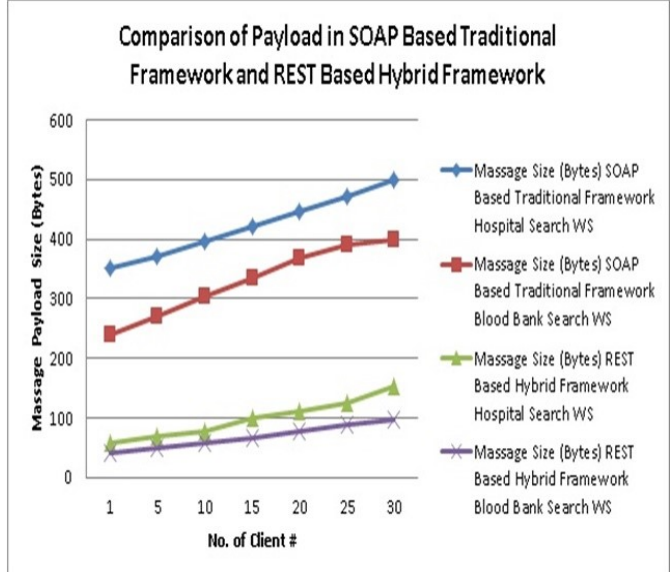

Figure 2: Payload Size Generated by SOAP and REST Based Hybrid Framework

Figure 3 shows a graphical representation of SOAP based traditional framework and REST based Hybrid framework for response time. The response time is measured for varying number of concurrent client requests.

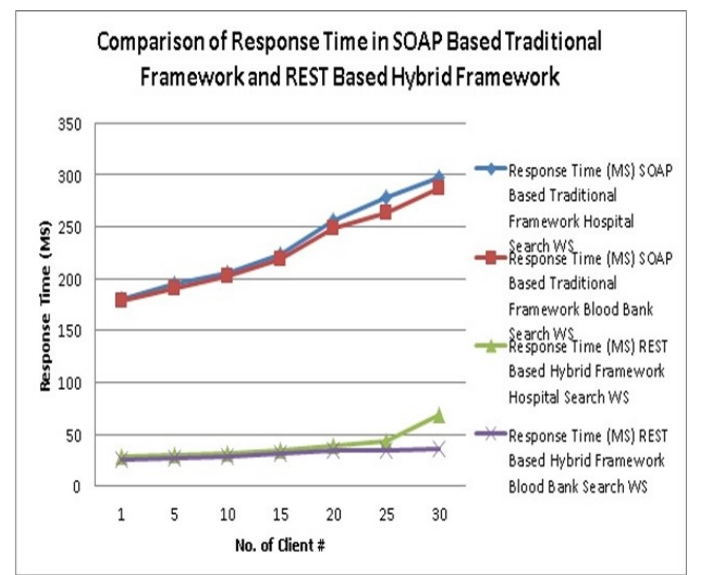

Figure 3: Response Time Required for SOAP and REST

\section{Performance Measure for Varying Load}

Performance of SOAP based Traditional Framework and REST based Hybrid framework is analyzed through measuring the effect of different request message size from $3 \mathrm{~KB}$ to $100 \mathrm{~KB}$ on the response time. Figure 4 shows the performance comparison of a mobile host for different load. 


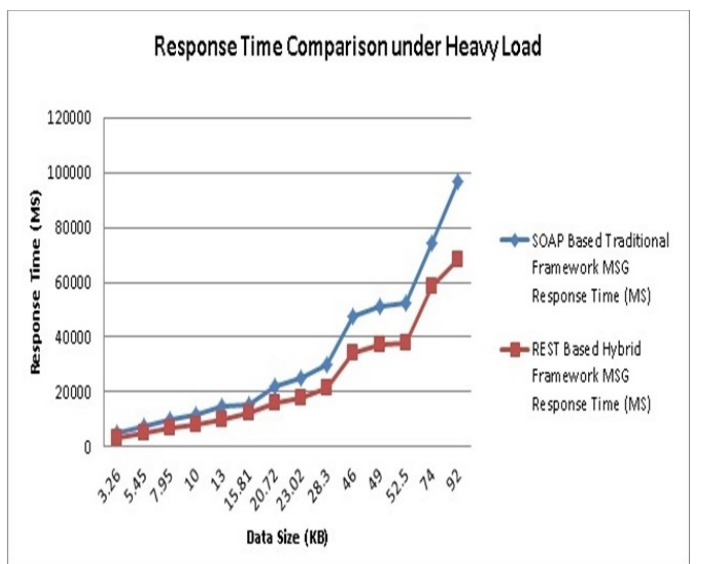

Figure 4: Performance of SOAP and REST on Different Load Size

\section{Performance Evaluation of Hybrid framework for SMS Users}

For testing the working of Hybrid framework for SMS clients, consider two scenarios. The first scenario is the Host and client are in the same area and second scenario is host and client is in different area. The response time measured in both scenarios and shown in figure 5.

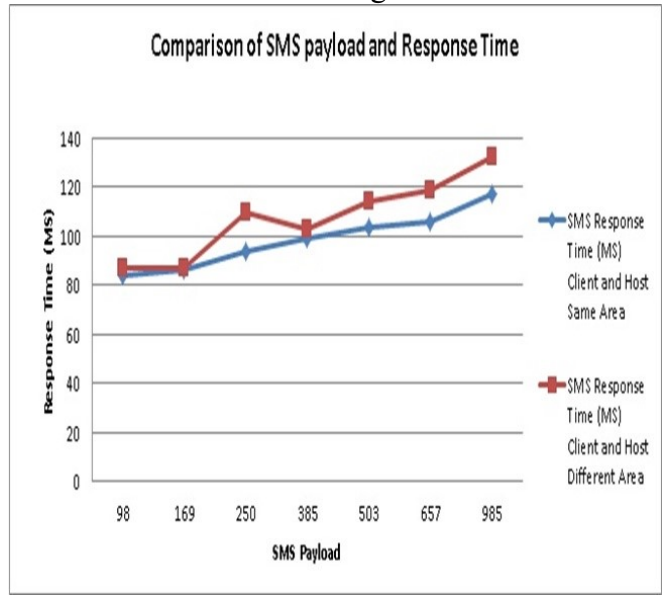

Figure 5: SMS Payload Comparison

Battery Consumption Analysis of Mobile Host

When deploying Host on Smart Phone it consumes very less battery. The observation of battery consumption of mobile host after deploying .apk file on the device along with its normal working of Smart phone. Figure 6 shows a graphical representation of Battery consumption with number of hours.

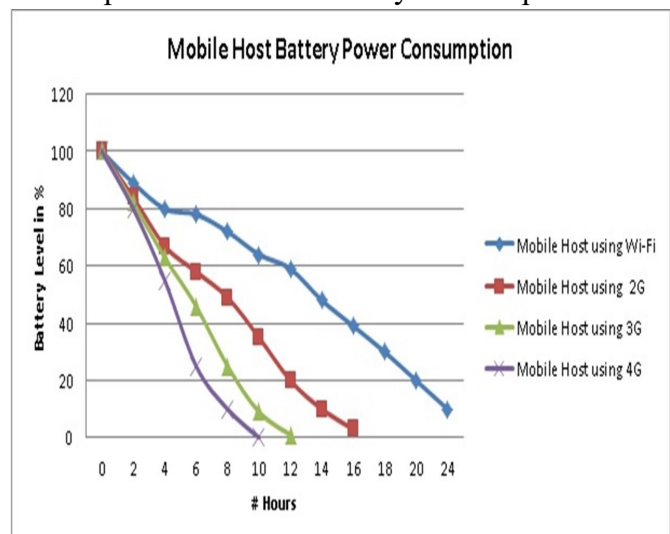

Figure 6: Battery Consumption of Different Wireless Networks 


\section{Conclusion}

It is feasible to deploy web services on android host due to the combination of technologies from the telecommunication, Information Technology, advances in mobile hardware. The use of mobile devices as web service provider is on the rise. To design a novel lightweight hybrid framework for mobile web service provisioning, we have developed the Hybrid framework and presented the details of the implementation. We chose Android and Java as the language platform for implementation of the framework. This framework prototype can run on mobile phones and tablets. More generally, the framework is a step toward to check the feasibility in the open source Android platform at the application level. The proof of concept in terms of feasibility, scalability, efficiency and availability has been achieved by analyzing the performance of mobile host. It was analyzed that from the payload generated by SOAP based web service framework is more as compared to REST based web service framework and this affects the response time. The response time is more in SOAP based web service frameworks because messages are wrapped with SOAP envelop and parser takes more time to parse the request. In REST based framework directly communicate with mobile host through HTTP and parser parse the request quickly. In the scalability analysis, the Mobile Host tested with concurrent access of user and this is compared with the traditional SOAP based framework. It was identified that REST based Hybrid framework is more scalable as compared to the conventional web service framework. When adding more concurrent requests to measure the threshold value, SOAP-based traditional framework starts to reject requests early as compared to REST based Hybrid Framework, SOAP-based consumes more resources as compared to REST. Thus, REST based Hybrid framework is more scalable and reliable than SOAP based traditional framework. This framework also tested for accessing mobile host through SMS messaging as an alternative and it's a better solution for those who can't afford wireless internet. The framework also supports alternative ways to access web services without mobile internet through SMS service and these services also work efficiently in low mobile network range areas too. The limitation of this prototype is the SMS cost and when response size is more than 160 characters, it sends a number of messages at a time. It is also providing quick response in low mobile range area. In future mobile host should be accessible through local languages.

\section{References}

1. Wagh, Kishor S., and Ravindra C. Thool. Performance Analysis of Mobile Web Service Provisioning On Different Mobile Host, Annual IEEE India Conference (INDICON), pp. 1-5, 2014.

2. Market prediction for mobile data obtained Cisco Visual Networking Index, http://www.cisco.com/c/en/us/solutions/collateral/service-provider/visual-networking-index-vni/white paper c11-520862.html/

3. Smart phone OS Market Share prediction for mobiles OS by IDC, http://www.idc.com/prodserv/smartphoneos-market-share.jsp/

4. S. Vinoski. (1997) 'CORBA: Integrating Diverse Applications Within Distributed Heterogeneous Environments', IEEE Communication Magazine, Vol. 35, No. 2, pp.46-55

5. J. Maassen, R. van Nieuwpoort, R. Veldema, H. E. Bal, and A. Plaat. (1999) 'An Efficient Implementation of Java's Remote Method Invocation', Seventh ACM SIGPLAN Symp. Princ. Pract. Parallel Program, Vol. 34, No. 8, pp.173-182

6. F. Plil and M. Stal. (1998) 'An Architectural View of Distributed Objects and Components in CORBA, Java RMI, and COM / DCOM', Software Concepts and Tools, Vol. 19, No. 1, pp.14-28

7. J. Ang and M. Luo. (2004) 'Patterns: Service- Oriented Architecture and Web Services', IBM Red-Book, International Technical Support Organization.

8. W. Lee, C. M. Lee, J. W. Lee, and J. S. Sohn. (2009) 'ROA Based Web Service Provisioning Methodology for Telco and its Implementation', Lecture Notes in Computer Science (including subseries Lecture Notes in Artificial Intelligence and Lecture Notes in Bioinformatics, pp.511-514

9. Roy Thomas Fielding. 2000 'Architectural Styles and the Design of Network-Based Software Architectures', PhD Thesis, UNIVERSITY OF CALIFORNIA, IRVINE.

10. L. Richardson and S. Ruby. (2007) 'RESTful Web Services', OReilly Media, First Edition. WSDL, http://www.w3.org/TR/wsd120/

11. J. Meng, S. Mei, and Z. Yan. (2009) 'RESTful Web Services: A Solution for Distributed Data Integration', International Conference on Computational Intelligence and Software Engineering, pp.1-4

12. F. Belqasmi and R. Glitho. (2011) 'RESTful Web Services for Service Provisioning in Next-Generation Networks: A Survey', IEEE Communications Magazine, Vol. 49, No. 12, pp.63-73

13. F. Belqasmi and R. Glitho. (2008) 'RESTful Web Services vs . Big Web Services: Making the Right Architectural Decision Categories and Subject Descriptors', Proceedings of the 17th World Wide Web Conference, Beijing, China, pp.805-814 
14. Fatna Belqasmi, Jagdeep Singh, Suhib Younis Bani Melhem, Roch H. Glitho. (2012) 'SOAP-Based vs. RESTful Web Services: A Case Study for Multimedia Conferencing', IEEE Internet Computing, Vol. 16, No.4, pp.54-63

15. F. Aijaz, S. Z. Ali, M. A. Chaudhary, and B. Walke. (2009) 'Enabling High Performance Mobile Web Services Provisioning', IEEE 70th Vehicular Technology Conference Fall (VTC 2009-Fall), pp.1-6

16. S. Allamaraju. (2010) 'RESTful Web Services', OReilly Media, First Edition. Roots of the REST/SOAP debate, http://conferences.idealliance.org/extreme/html/2002/Prescod01/EML2002Prescod01.html

17. S. N. Srirama, M. Jarke, S. Augustin, W. Prinz, R. (2007) 'Mobile Web Services Mediation Framework', Proceedings of the 2nd Workshop on Middleware for Service Oriented Computing, pp.6-11.

18. Srirama, Satish Narayana and Jarke, Matthias and Prinz, Wolfgang. (2008) 'MWSMF: A Mediation Framework Realizing Scalable Mobile Web Service Provisioning', Proceedings of the 1st International Conference on Mobile Wireless Middleware, pp.1-7.

19. Srirama, S.; Vainikko, E.; or, V.; Jarke, M. (2010) 'Scalable Mobile Web Services Mediation Framework', Fifth International Conference on Internet and Web Applications and Services (ICIW), pp.315320.

20. Yeon-Seok Kim and Kyong-Ho Lee. (2007) 'A Lightweight Framework for Hosting Web Services on Mobile

Devices', Fifth European Conference on Web Services (ECOWS07), pp.255-263.

21. Yeon-Seok Kim and Kyong-Ho Lee. (2009) 'A Lightweight Framework for Mobile Web Services', Journal of Computer Science - Research and Development(Springer-Verlag), vol. 24, no. 4, pp. 199-209.

22. M. Asif and S. Majumdar. (2011) 'A Runtime Partitioning Technique for Mobile Web Services', 40th International Conference on Parallel Processing Workshops (ICPPW), pp. 81-90.

23. Asif M. and S. Majumdar. (2011)' Partitioning Frameworks for Mobile Web Services Provisioning', International Journal of Parallel, Emergent and Distributed Systems, Vol. 26, No. 6, pp. 519-544.

24. K. Elgazzar, P. Martin, and H. Hassanein. (2012) 'A Framework for Efficient Web Services Provisioning in Mobile Environments', Mobile Computing, Applications, and Services, Springer Berlin Heidelberg, pp. $246-262$.

25. K. Elgazzar, H. S. Hassanein, and P. Martin. (2013) 'Towards personal mobile Web services', IEEE Wireless Communications and Networking Conference (WCNC), pp. 4606-4611.

26. K. Wagh, Dr. R. Thool, "Mobile Web Service Provisioning and Performance Evaluation of Mobile Host," International Journal on Web Service Computing, Vol. 5, No. 2, June 2014. 